\title{
Dividing sets as nodal sets of an eigenfunction of the Laplacian
}

\author{
SAMUEL T LISI
}

\begin{abstract}
We show that for any convex surface $S$ in a contact 3-manifold, there exists a metric on $S$ and a neighbourhood contact isotopic to $S \times I$ with the contact structure given by $\operatorname{ker}(u d t-\star d u)$ where $u$ is an eigenfunction of the Laplacian on $S$ and $\star$ is the Hodge star from the metric on $S$. This answers a question posed by Komendarczyk [3].
\end{abstract}

57R17; 53D10

Given a convex surface $S$ in a contact 3-manifold, we show the existence of a metric defined on a tubular neighbourhood of $S$, adapted to the contact structure, for which the dividing curves are nodal curves of an eigenfunction of the Laplacian on $S$. In our construction, we show that any dividing set may be realized in this way. This addresses two questions raised by Komendarczyk [3].

Definition 1 Let $(M, \xi)$ be a contact 3-manifold with co-oriented contact structure $\xi$. A metric $g$ on $M$ is adapted to the contact structure if there exists a contact form $\alpha$ generating the contact structure so that $\star \alpha=d \alpha$.

A class of examples of metrics adapted to a contact structure $\xi=\operatorname{ker} \alpha$ is given by taking an almost complex structure $J$ on $\xi$ compatible with $d \alpha$, ie so that $d \alpha(\cdot, J \cdot)$ is a metric on $\xi$. Then, we construct a metric adapted to $\xi$ by taking $g=\alpha^{2}+d \alpha(\cdot, J \cdot)$.

Definition 2 (Convex surface; see Giroux [2]) A surface $S$ in a contact 3-manifold $(M, \xi)$ is convex if there exists a (local) vector field $\mathbf{v}$ transverse to $S$ so that $L_{\mathbf{v}} \xi=0$.

The dividing set is the set of all points on $S$ where $\mathbf{v} \in \xi$. (The contact condition forces this to be an embedded multicurve in $S$.)

The dividing set divides the surface $S$ into two open submanifolds, $S_{+}$on which $\mathbf{v}$ is positively transverse to $\xi$ and $S_{-}$on which $\mathbf{v}$ is negatively transverse to $\xi$.

Our main result can then be stated as follows: 
Theorem 3 Let $S$ be a convex surface in the co-oriented contact 3-manifold $(M, \xi)$. Then, there exist an isotopic surface $S^{\prime}$, an adapted metric $g$ and an eigenfunction $u$ of $\left.\triangle_{g}\right|_{S^{\prime}}$ so that a neighbourhood of $S^{\prime}$ is contactomorphic to $S^{\prime} \times I$ with the contact structure

$$
\operatorname{ker}(u d t-\star d u) \text {. }
$$

Furthermore, this metric $g$ may be taken to be $t$-translation invariant in the neighbourhood of $S^{\prime}$.

(Here, we take the conventions $\alpha \wedge \star \alpha=|\alpha|_{g}^{2} d$ vol, and $\triangle_{g} u=\star d \star d u$.)

Komendarczyk [3; 4] proved this result, first in the case the dividing set is an embedded circle, and then generalizing it to the case in which the complement of the dividing set consists of two connected components, by using techniques from spectral geometry. In recent personal communication, he has explained to the author a possible extension of these methods to the general case. In contrast to his methods, we prove the result using "soft" techniques in contact topology.

The relationship between adapted metrics and contact topology has just recently begun to be exploited, notably by Etnyre, Massot and Komendarczyk [1].

The main interest of convex surfaces in contact topology comes from Giroux's flexibility theorem [2]:

Theorem 4 Suppose $\Sigma$ is a closed convex surface in $(M, \xi)$, with transverse contact vector field $\mathbf{v}$ and dividing curves $\Gamma$. Suppose $\mathcal{F}$ is a singular foliation on $\Sigma$ divided by $\Gamma$. Then, there exists an isotopy $\phi_{s}, s \in[0,1]$, so that $\phi_{0}(\Sigma)=\Sigma,\left.\xi\right|_{\phi_{1}(\Sigma)}=\phi_{1}(\mathcal{F})$, $\phi$ fixed on $\Gamma$ and $\phi_{s}(\Sigma)$ transverse to $\mathbf{v}$ for all $s$.

Heuristically speaking, this tells us that the neighbourhood of $S$ is described, up to isotopy, by the dividing curves. In particular, if $\Sigma \times \mathbb{R}$ admits two translation invariant contact structures giving the same dividing curves on $S$ and cutting out the same $S_{ \pm}$ regions, then the two contact structures are isotopic.

The proof of Theorem 3 will be by constructing the metric and the eigenfunction, and will exploit the soft aspects of symplectic and contact topology. Instead of constructing these directly, we will construct a symplectic form on $S$ and an almost complex structure compatible with it. Reformulated in this way, we obtain:

Theorem 5 Let $S$ be a closed, connected surface, and consider the $t$-invariant contact structure on $\mathbb{R} \times S$ given by $\xi_{0}=\operatorname{ker}\left(\alpha_{0}=f d t+\beta\right)$. Denote the dividing curves by $\Gamma=f^{-1}(0) \subset S$. 
Orient $S$ by $i_{\partial_{t}}\left(\alpha_{0} \wedge d \alpha_{0}\right)$. Then, there exist an area form $\Omega$ on $S$, compatible with the orientation, a compatible complex structure $j$, and a function $u: S \rightarrow \mathbb{R}$ with

$$
d(d u \circ j)=u \Omega \quad \text { and } \quad u^{2}+|d u|^{2}>0,
$$

and so that $u^{-1}(0)=f^{-1}(0)$, and so the contact form $u d t+d u \circ j$ induces the same $S_{+}$and $S_{-}$regions.

Proof of Theorem 3 We will now show that Theorem 5 implies Theorem 3.

Let $S$ be a convex surface in $(M, \xi)$ with transverse contact vector field $\mathbf{v}$. Let $\Gamma$ be the dividing set. Then, by following the flow of $\mathbf{v}$, there exists a neighbourhood of $S$ in $M$ contactomorphic to a neighbourhood of $\{0\} \times S$ in $\mathbb{R} \times S$, with contact structure given by the contact form

$$
\alpha_{0}=f d t+\beta,
$$

where $f$ and $\beta$ are a function and a one-form on $S$ respectively. Then, $f^{-1}(0)=\Gamma$.

Let $u, j$ and $\Omega$ be as in Theorem 5 . This then gives a contact form on $\mathbb{R} \times S$ by $\alpha_{1}=u d t+d u \circ j$. Define a metric on $\mathbb{R} \times S$ by setting $g=d t^{2}+\Omega(\cdot, j \cdot)$. We now observe $\left.\triangle_{g}\right|_{S} u \Omega=-d(d u \circ j)$, so $u$ as in Theorem 5 indeed is an eigenfunction of the Laplace operator of $g$ restricted to $S$. It now remains to verify that this metric $g$ is adapted to the contact structure. We observe that $d \operatorname{vol}_{g}=d t \wedge \Omega$ and that $g\left(\partial_{t}, \cdot\right)=d t, g\left(-X_{u}, \cdot\right)=d u \circ j$ and $g\left(-j X_{u}, \cdot\right)=d u$. It follows that $\star d t=\Omega$ and $\star d u \circ j=-d t \wedge d u$. Thus,

$$
\star(u d t+d u \circ j)=u \Omega-d t \wedge d u=d \alpha_{1} .
$$

as required.

We now have two translation invariant contact structures on $\mathbb{R} \times S$, generated by the contact forms $\alpha_{0}$ and $\alpha_{1}$. The dividing sets and induced orientations on $S$ are the same, so by Giroux's Flexibility Theorem, the contact structures are contact isotopic. Following the image of the isotopy in $M$, and restricting to a sufficiently small interval around $t=0$ gives the resulting $S^{\prime}$. This now constructs a local contact metric, defined in a neighbourhood of $S$.

In order to extend this local metric to a global one, first extend the contact form to a global contact form on $M$. Note the space of contact metrics is nonempty and fibrewise convex. Now interpolate between our locally defined metric and an arbitrary global one to obtain a globally defined contact metric with the desired properties.

The main result, Theorem 5, is a corollary of the following result: 
Proposition 6 Let $S$ be a closed, connected surface and $\Gamma \subset S$ be a collection of embedded circles dividing $S$ into two regions, so that

$$
S \cong S_{-} \cup([-1,1] \times \Gamma) \cup S_{+},
$$

where $S_{ \pm}$are two open submanifolds of $S$. Then, there exist a smooth function $u: S \rightarrow \mathbb{R}$, an area form $\Omega$ and a compatible complex structure $j$ on $S$ so that

$$
\begin{aligned}
d(d u \circ j) & =u \Omega \\
u^{-1}(0) & =\Gamma \\
u^{2}+|d u|^{2} & >0 .
\end{aligned}
$$

The remainder of this paper is a proof of Proposition 6 .

\section{Proof of Proposition 6}

The key step in the proof of Proposition 6 is the following Lemma, whose proof will come later.

Lemma 7 Let $S=S_{-} \cup([-1,1] \times \Gamma) \cup S_{+}$as in the hypothesis of Proposition 6 . There exist an area form $\omega$, a compatible complex structure $j$, and a real valued function $F$ on $S$ so that for some constants $C>0$ and $\epsilon>0$ the following properties hold:

(i) $\max _{S}|F|<\pi / 2, F^{-1}(0)=\{0\} \times \Gamma \subset[-1,1] \times \Gamma$,

(ii) $F<0$ on $S_{-} \cup([-1,0) \times \Gamma), F>0$ on $((0,1] \times \Gamma) \cup S_{+}, d F \neq 0$ on $[-1,1] \times \Gamma$,

(iii) $d(d F \circ j) \leq 0$ on $[-1,0] \times \Gamma$ and $d(d F \circ j)<0$ on $S_{-}$,

(iv) $d(d F \circ j) \geq 0$ on $[0,1] \times \Gamma$ and $d(d F \circ j)>0$ on $S_{+}$,

(v) for $(s, t) \in(-\epsilon, \epsilon) \times \Gamma, F(s, t)=C s$ and $\omega=d s \wedge d t$.

Proof of Proposition 6 Let $F, j$ and $\omega$ be as in Lemma 7. Define a real valued function on $S$ by

$$
u=\sin (F) .
$$

Define $\triangle F$ by $-(\triangle F) \omega=d(d F \circ j)$. Then, we obtain

$$
\begin{aligned}
d(d u \circ j) & =u|d F|^{2} \omega+\cos (F) d(d F \circ j) \\
& =u\left(|d F|^{2}-\frac{\cos (F)}{\sin (F)} \triangle F\right) \omega .
\end{aligned}
$$


Observe that from the definition of $F, \triangle F=0$ on $(-\epsilon, \epsilon) \times \Gamma$. Since $|F|<\pi / 2$, $\sin (F)$ has the same sign as $F$. Thus, $-(1 / \sin (F)) \triangle F \geq 0$ is nonsingular, and only vanishes in a subset of $[-1,1] \times \Gamma$, where $d F$ is nonvanishing. Hence,

$$
|d F|^{2}-\frac{\cos (F)}{\sin (F)} \triangle F>0 .
$$

Thus, by taking

$$
\Omega=\left(|d F|^{2}-\frac{\cos (F)}{\sin (F)} \triangle F\right) \omega
$$

we obtain a volume form on $S$ so that $d(d u \circ j)=u \Omega$. We claim this triple of $u, \omega$ and $j$ has the desired properties.

Since $|F|<\pi / 2$, it follows that $u>0$ on $((0,1] \times \Gamma) \cup S_{+}$, and that $u<0$ on $S_{-} \cup([-1,0) \times \Gamma)$. Furthermore, $u^{-1}(0)=F^{-1}(0)=\{0\} \times \Gamma$.

To show $u^{2}+|d u|^{2}>0$, it suffices to check near $u^{-1}(0)=\{0\} \times \Gamma$. Note, however, that in a neighbourhood of $\{0\} \times \Gamma, F(s, t)=C s$, for some positive constant $C$, and thus $d u=\cos (F) d F=\cos (C s) C d s$, which is nonvanishing in a neighbourhood of $\{0\} \times \Gamma$. This completes the proof of Proposition 6 .

We now prove the key Lemma 7. This involves constructing a weakly subharmonic function on each of $S_{+}$and $S_{-}$, strictly subharmonic away from the dividing curves, but harmonic near the boundary.

Proof of Lemma 7 Observe first that $S_{+}$and $S_{-}$admit Stein structures since they are open Riemann surfaces, ie they each admit exhausting strictly subharmonic functions, for which the boundary of $S_{ \pm}$is a level set. Furthermore, recall that $\partial S_{+}=\Gamma=\partial S_{-}$, with opposite orientations. We now apply the following Lemma (whose proof we defer) to each of $S_{+}$and $S_{-}$.

Lemma 8 Let $(\Sigma, j)$ be a compact Riemann surface with boundary.

Suppose $f: \Sigma \rightarrow \mathbb{R}$ is bounded below, $f^{-1}(-1)=\partial \Sigma$ and $-d(d f \circ j)=\omega_{0}$ is an area form compatible with $j$. (By the maximum principle, it follows that $f$ is bounded above by -1.)

Then, with $\tilde{\Sigma}=\Sigma \cup([-1,0] \times \partial \Sigma)$, there exist $\epsilon>0$, a nonpositive smooth function, $g: \widetilde{\Sigma} \rightarrow(-\infty, 0]$, an extension of $j$ to $\widetilde{\Sigma}$, and a volume form $\omega$ on $\widetilde{\Sigma}$ compatible 
with $j$, with the following properties:

$$
\begin{gathered}
j=i \text { on }[-1,0] \times \Sigma \\
g=f \text { on } \Sigma \\
g<0 \text { on } \Sigma \cup([-1,0) \times \partial \Sigma) \\
\left.g\right|_{[-\epsilon, 0] \times \partial \Sigma:(s, t) \mapsto 2 s} \\
\left.\omega\right|_{[-\epsilon, 0] \times \partial \Sigma}=d s \wedge d t \\
-d(d g \circ j) \geq 0 \text { on } \Sigma \cup([-1,0] \times \partial \Sigma) \\
-d(d g \circ j)=\omega \text { on } \Sigma .
\end{gathered}
$$

Let $g_{ \pm}$be the (weakly) subharmonic functions, and let $\omega_{ \pm}$be the area forms from Lemma 8.

Define the following function on $S=S_{-} \cup([-1,1] \times \Gamma) \cup S_{+}$by

$$
F= \begin{cases}g_{-} & \text {on } S_{-} \cup\left([-1,0] \times \partial S_{-}\right), \\ -g_{+}(-s, t) & \text { on }[0,1] \times \partial S_{+}, \\ -g_{+} & \text {on } S_{+} .\end{cases}
$$

Note that $F$ then defines a smooth function on $S$, since for some $\epsilon>0, g_{-}(s, t)=2 s$ for $(s, t) \in[-\epsilon, 0] \times \partial S_{-}$and $-g_{+}(-s, t)=2 s$ for $(s, t) \in[0, \epsilon] \times \partial S_{+}$. Furthermore, the area form defined by

$$
\omega= \begin{cases}w_{-} & \text {on } S_{-} \cup\left([-1,0] \times \partial S_{-}\right), \\ w_{+} & \text {on }\left([0,1] \times \partial S_{+}\right) \cup S_{+}\end{cases}
$$

is a smooth area form on $S$.

It then follows that

$$
\begin{array}{rlrl}
d(d F \circ j) & =-\omega & \text { on } S_{-} \\
& \leq 0 & & \text { on } S_{-} \cup\left([-1,0] \times \partial S_{-}\right) \\
& =0 & & \text { on }[-\epsilon, \epsilon] \times \Gamma \\
& \geq 0 & & \text { on }\left([0,1] \times \partial S_{+}\right) \cup S_{+} \\
& =\omega & & \text { on } S_{+} .
\end{array}
$$

By scaling $F$, we may set $|F|<\pi / 2$. Furthermore, by construction, $F(s, t)=C s$ for $s$ close to 0 in $[-1,1] \times \Gamma$, and $C>0$ a constant.

We now present the proof of Lemma 8. This uses the fact that the Stein structure on $\Sigma$ may be extended smoothly to a cylindrical end glued at the boundary. (Recall that a 
Stein structure consists of the data of a complex structure and an exhausting Morse function, plurisubharmonic with respect to the complex structure.) We then deform the standard model of the cylindrical end to obtain the desired weakly subharmonic function to have linear growth at the end. In essence, this deformation smoothes a strictly monotone, piecewise-smooth, weakly convex function on $\mathbb{R}$ to obtain a smooth, weakly convex function with a prescribed zero.

Proof of Lemma 8 Denote by $\partial_{k} \Sigma, k=1, \ldots, N$, the components of the boundary $\partial \Sigma$. First, complete $\Sigma$ by gluing the cylinder $[-1,+\infty) \times S^{1}$ to each boundary component $\partial_{k} \Sigma$. Denote each of these cylinders by $Z_{k}$.

Now extend the (strictly) subharmonic function $f$ to $Z_{k}$ by the function given in the cylinder coordinates by

$$
f_{k}(s, t)=A_{k}\left(\mathrm{e}^{s}-1 / \mathrm{e}\right)-1
$$

and extend the complex structure to the cylinders by $i$. Note that the resulting function is only continuous, and piecewise differentiable. For each $k$, by choosing the constant $A_{k}$ sufficiently large, the normal derivative of $f_{k}$ from the left at $s=-1$ may be arranged to be strictly less than the normal derivative of $f_{k}$ from the right. Thus, this continuous function satisfies the mean-value-inequality on $\Sigma \cup([-1, \infty) \times \partial \Sigma)$. Convolving with an approximate identity will give a smooth function, subharmonic if sufficiently $C^{0}$ close to the original function, and equal to the original function outside of a sufficiently small neighbourhood of $\partial \Sigma$. We may therefore take $f$ to be smooth.

This then extends the symplectic form by $\omega_{0}=A_{k} \mathrm{e}^{s} d s \wedge d t$. Denote these extensions again by $f, j$, and $\omega_{0}$, which are now defined on $\Sigma \cup([-1, \infty) \times \partial \Sigma)$. By scaling $f$ (and thus $\omega_{0}$ ) as necessary, we may assume $A_{k}<\frac{1}{2}$.

We now claim that for each $k$, there exists a function $G_{A_{k}}:[-1,1] \rightarrow \mathbb{R}$ with the following properties:

(1) $G_{A_{k}}^{\prime}(s)>0$ for all $s \in[-1,1]$,

(2) $G_{A_{k}}^{\prime \prime}(s) \geq 0$ for all $s \in[-1,1]$,

(3) $G_{A_{k}}(s)=A\left(\mathrm{e}^{s}-1 / \mathrm{e}\right)-1$ for $s$ near -1 ,

(4) $G_{A_{k}}(s)=2 s$ for $s$ near 0 .

To construct such a function $G_{A_{k}}$, for each $k$, we choose a monotone increasing function $\beta:[-1,1] \rightarrow[0,1]$ for which $\beta(s)=0$ for $s$ near -1 and $\beta(s)=1$ for $s$ near 0 . We then define

$$
G_{A_{k}}(s)=\int_{0}^{s}\left(1-\beta\left(s^{\prime}\right)\right) A_{k} \mathrm{e}^{s^{\prime}}+\beta\left(s^{\prime}\right) 2 d s^{\prime} .
$$


For a correct choice $\beta$, we may obtain that $G_{A_{k}}(-1)=-1$. From this, it follows that $G_{A_{k}}$ has the required properties.

Introduce the following function, $g$ defined on $\Sigma \cup([-1,0] \times \partial \Sigma)$ by

$$
g= \begin{cases}f & \text { on } \Sigma, \\ G_{A_{k}} & \text { on }[-1,0] \times \partial_{k} \Sigma .\end{cases}
$$

Then, observe

$$
d(d g \circ j)= \begin{cases}-\omega_{0} & \text { on } \Sigma, \\ -G_{A_{k}}^{\prime \prime}(s) d s \wedge d t & \text { on }[-1,0] \times \partial_{k} \Sigma .\end{cases}
$$

Also note that in any of the $(s, t)$ coordinates near $\partial_{k} \Sigma, g(s, t)=2 s$ for $s$ sufficiently close to 0 .

Define

$$
\omega= \begin{cases}\omega_{0} & \text { on } \Sigma, \\ \mu_{k}(s) d s \wedge d t & \text { on }[-1,0] \times \partial_{k} \Sigma,\end{cases}
$$

where $\mu_{k}(s)>0$, with the properties that $\mu_{k}(s)=A_{k} \mathrm{e}^{s}$ for $s$ near \pm 1 and $\mu_{k}(s)=1$ for $s$ near 0 . Therefore, from Equation (3), there exists a nonnegative function $K: \Sigma \cup([-1,0] \times \partial \Sigma) \rightarrow \mathbb{R}$ so that

$$
d(d g \circ j)=-K \omega .
$$

Furthermore, $K=1$ on $\Sigma$ and $K=0$ for $s$ near 0 in $[-1, \infty) \times \partial \Sigma$. This therefore constructs the desired $g$ and $\omega$.

Acknowledgements I would like to thank Richard Siefring and Joe Coffey for suggesting this problem to me, Ko Honda for helpful discussions and encouragement, and Paolo Ghiggini for both encouragement and helpful comments. I would like to thank Jorge Moraleda for useful discussions, and the referee for helpful suggestions.

\section{References}

[1] J Etnyre, R Komendarczyk, P Massot, Contact metric manifolds and tight contact structures arXiv:0906.3487v2

[2] E Giroux, Convexité en topologie de contact, Comment. Math. Helv. 66 (1991) 637-677 MR1129802

[3] R Komendarczyk, On the contact geometry of nodal sets, Trans. Amer. Math. Soc. 358 (2006) 2399-2413 MR2204037 
[4] R Komendarczyk, Nodal sets and contact structures, $\mathrm{PhD}$ thesis, Georgia Institute of Technology (2008) Available at http://hdl.handle.net/1853/11517

Département de Mathématique, Université Libre de Bruxelles, CP 218

Boulevard du Triomphe, B-1050 Bruxelles, Belgium

Samuel.Lisi@ulb.ac.be

http://homepages.ulb.ac.be/ samulisi/

Received: 1 April 2010 Revised: 3 November 2010 